\title{
ON THE REDUCING ACTION OF HYDROGEN. (Preliminary Note.)
}

\author{
By ALFRED C. CHAPMAN AND H. D. LAW.
}

Notwithstanding the great theoretical and practical importance attaching to a more intimate knowledge of the properties of the hydrogen evolved during the solution of metals in acids, very little attention appears to have been devoted to this subject until quite recently, the employment of the expressions " nascent " and "free " being apparently considered sufficient to cover all the known facts. If the conceptions of atomic or active and molecular or inactive hydrogen were all-sufficient, the source of the hydrogen in any reduction process should be a matter of indifference so far as the reducing action is concerned; but yet it is quite well known that this is by no means the case, and that the reducing agent to be employed must be chosen in careful relation to the reduction which it is desired to bring about. This is well seen in connection with the Marsh-Berzelius test for arsenic, for not only does the hydrogen formed at the surface of a platinum cathode in the electrolytic method fail to reduce arsenates, but quite different results may be obtained in the zinc method by the employment of various samples of zinc. Before alluding to the results of our experiments, we may perhaps be permitted to refer very briefly to the following elementary theoretical considerations :

When a rod of zinc is immersed in dilute sulphuric acid, the zinc of course tends to pass into solution and to "force out" the hydrogen. On the other hand, a hydrogen electrode placed in a solution of zinc sulphate tends to cause the deposition of metallic zinc. Thus in the case we are considering (dilute sulphuric acid and zinc) hydrogen will be liberated only when the solution tension of the metal is greater 
than that of hydrogen. The solution tension of hydrogen is about 1 volt, whilst that of zinc is about 1.7 volts, so that an evolution of hydrogen might be expected. There is, however, another important factor which comes into play-viz., the "retaining" effect which every metal exerts on the hydrogen formed at its surface-and so in many cases a potential far in excess of that of the hydrogen electrode is necessary before any free hydrogen is obtained-that is, before the hydrogen is liberated. This excess of potential is known as "supertension," and in the case of zinc amounts to about 0.7 volt above that of the hydrogen electrode. From this it will be seen that in the case of pure zinc and sulphuric acid little or no evolution of hydrogen is to be expected. Other metals, however-e.g., platinum-have smaller supertensions, and consequently small quantities of such metals deposited on the surface of the pure zinc will allow the gas to come off freely and at a lower potential, its activity being in consequence sometimes reduced to a point which renders it incapable of bringing about the reduction of difficultly reducible substances. The application of these considerations to the explanation of the insensitiveness of zinc in the MarshBerzelius test will, we think, be obvious. The metal, as used in our laboratories, is necessarily more or less impure, frequently containing particles of metals of low supertension-such, for example, as iron. Hydrogen is therefore liberated rapidly, and at a potential too low to permit of the reduction of arsenious acid to arsenic hydride under the conditions of the experiment. Thus we have been able to show that such low supertension metals as palladium, platinum, copper, nickel, cobalt, silver, and iron all render zinc insensitive, whilst cadmium, lead, and tin, which are possessed of high supertensions, have no effect on the reducing power of sensitive zinc. Starting with a sensitive sample of this metal, we have been able to render it exceedingly insensitive by depositing on its surface small quantities of iron, cobalt, etc.; and, conversely, we have succeeded in rendering the highly insensitive metal fully sensitive by the use of metals of high supertension. In the near future we hope to publish a detailed account of our work in this field. Throughout the investigation the Marsh-Berzelius arsenic process has been used merely as a convenient and delicate method of determining the amount of reduction taking place, and in a future paper we hope to extend the inquiry to other and widely differing substances.

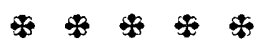

